\title{
Medical system error causing methotrexate poisoning and delay in diagnosis: report of two cases
}

\author{
Hamidreza Reihani ${ }^{1}$, Maryam Ziadi Lotfabadi $^{*}$ \\ 'Department of Emergency Medicine, Faculty of Medicine, Mashhad University of Medical Sciences, Mashhad, Iran \\ 2Department of Emergency Medicine, Mashhad University of Medical Sciences, Mashhad, Iran
}

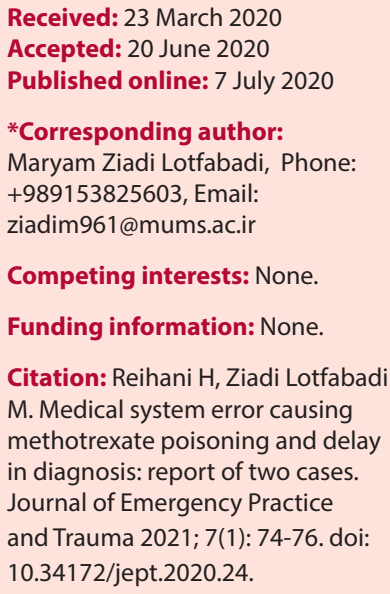

\begin{abstract}
Objective: We are going to alert the emergency physicians to be aware of drugs side effects. Some drugs like methotrexate are not routine drugs, and the symptoms may be miss diagnosed if not completely notified.

Case Presentation: We present two patients under treatment with methotrexate. Both of them suffered from methotrexate poisoning due to improper use. We discuss the causes of methotrexate poisoning and its prevention.

Conclusion: A significant proportion of methotrexate users may not be completely aware of its proper use and the side effects. One of the important ways to prevent the toxicity of methotrexate is educating patients and their companions in order to use it correctly.

Keywords: Methotrexate toxicity, Mucocutaneous lesions, Pancytopenia
\end{abstract}

\section{Introduction}

Drug side effects are one of the problems that an emergency medicine specialist should always keep in mind. Due to increased chronic diseases and increased use of methotrexate, methotrexate poisoning is more serious than ever. Methotrexate is an antifolate metabolite that inhibits dihydrofolate reductase (DHFR). The major manifestations of MTX toxicity are severe mucositis, bone marrow depression, renal and liver dysfunction $(1,2)$. MTX toxicity can occur because of accidental ingestion/overdose by the patient or due to prescription error. Methotrexate poisoning is usually caused by parenteral overdose or repeated chronic drug ingestion (1), but in our patients, high and chronic daily ingestion of the weekly dose caused clinically significant toxicity. Lack of careful attention to the drug history can be very misleading. We present two patients who had started treatment with methotrexate due to scleroderma and rheumatoid arthritis. Both of our patients suffered from methotrexate poisoning owing to improper use. In this article, we discuss the causes of methotrexate poisoning and its prevention.

Case Presentation

One of our patients was a 56-year-old woman with hypertension, diabetes and rheumatoid arthritis who had been taking methotrexate for three months. She had oral ulcers for two months which gradually intensified and became hemorrhagic. This condition had an effect on the patient nutrition as well. Our patient was hospitalized a week ago because of gastrointestinal bleeding and melena. Endoscopy was performed for the patient and the endoscopic report was gastroesophageal reflux disease (GERD). Then, she was referred to our center to investigate the cause of pancytopenia. The patient's laboratory results were: PLT: 76000/ $\mu \mathrm{L}, \mathrm{Hb}: 11.8 \mathrm{~g} / \mathrm{dL}$, and WBC: $1600 / \mu \mathrm{L}$. At the time of admission, our patient complained of generalized weakness, dry tongue, bleeding mouth ulcers, hematemesis, and melena. Other patient medications used included insulin, dorocontin, carvedilol, indomethacin and losartan. Methotrexate had started three months earlier. The methotrexate used by the patient was from two different drug companies (Figure 1). The prescription on the medicine box was as follows: One day a week, two tablets together and on the other box: Fridays, two tablets. The patient was instructed to consume two tablets of MTX in one day each week, however; the day was not determined. Our patient used both models of methotrexate tablets from two different drug companies. From one model, two tablets every day and from another 


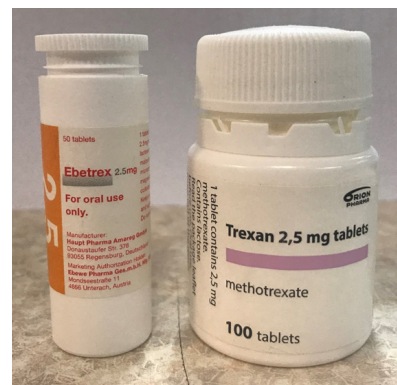

Figure 1. MTX tablets, two different models from two drug companies.

model, two tablets every Friday. At the time of referral, the patient's vital signs and symptoms were: blood pressure (BP): 100/60 mm Hg, pulse rate (PR): 85/min, and respiratory rate $(\mathrm{RR}): 15 / \mathrm{min}$. Also, the conjunctiva was pale. She had glossitis and multiple hemorrhagic lesions on the lips and palates. The patient's abdomen was soft and had hepatosplenomegaly. Abdominal and pelvic ultrasound revealed free fluid in Morrison and Interloop space. The ankle joints had pitting edema. During treatment, methotrexate was discontinued. The patient was treated with acyclovir, fluconazole, meropenem and vancomycin. Granulocyte-colony stimulating factor (GCSF) was injected. During hospitalization, oral lesions improved. Blood cell counts were normalized and the patient was discharged with a good general condition. At the first hospital where the patient was hospitalized, the patient's medication profile was not taken properly and methotrexate poisoning was remained undiagnosed.

The second patient was an 82-year-old man who had been treated with methotrexate for ten days due to skin lesions. The prescription written on the box was: first week, three tablets, and then weekly six tablets. However, week day was not specified and our patient mistakenly took 6 tablets every day. He had pancytopenia and was referred to our center to investigate the cause of gastrointestinal bleeding. On arrival, the patient had multiple oral lesions in the form of vesicles and bleeding ulcers that had started 4 days ago, and generalized rash was seen on the abdomen, upper and lower limbs (Figure 2A-2C). The patient had shock state. He complained of nausea and vomiting. Laboratory tests that were done at the previous center were: Platelet
(PLT): 19000/ $\mu \mathrm{L}$, hemoglobin $(\mathrm{Hb}): 11 \mathrm{~g} / \mathrm{dL}$, and white blood cell (WBC): 1200/ $\mu \mathrm{L}$. Lab test results in the course of hospitalization included: PLT: $6000 / \mu \mathrm{L}, \mathrm{Hb}: 10.2 \mathrm{~g} /$ dL, WBC: $800 / \mu \mathrm{L}$, alanine aminotransferase (ALT): 116 $\mathrm{U} / \mathrm{L}$, aspartate aminotransferase (AST): $153 \mathrm{U} / \mathrm{L}$, and alkaline phosphatase (ALP): $120 \mathrm{U} / \mathrm{L}$. Abdominal and pelvic ultrasound showed dilated common bile duct with a diameter of $22 \mathrm{~mm}$. Mild ectasia was also reported in intrahepatic bile ducts. Protective cares for the patient began. Serum methotrexate level was checked. Due to high blood levels of methotrexate, methotrexate was discontinued immediately. Pentazole and folinic acid were administered. The patient received 2 units of PRBCs and one vial of bicarbonate per liter of serum. During hospitalization, GCSF, acyclovir and fluconazole were prescribed. The oral ulcers healed, melena was not repeated and the patient was discharged by normal cell blood count.

\section{Discussion}

Methotrexate is an antifolate metabolite that inhibits DHFR. Conversion of folate to dihydrofolate and dihydrofolate to tetrahydrofolate decreases with methotrexate use that is needed for Dena synthesis $(2,3)$. This is the basis for the treatment of acute methotrexate toxicity (2). Since the first- pass metabolism of methotrexate, $80 \%$ to $90 \%$ is excreted unchanged in the urine, so, the evaluation of renal function before and during methotrexate treatment is necessary. Nephrotoxicity is caused by the deposition of methotrexate in the renal tubules at low $\mathrm{PH}$. Therefore, hydration and treatment with intravenous bicarbonate are recommended in order to get urine $\mathrm{pH}$ higher than $8(2,3)$. A number of patient-related factors increase the risk of acute kidney injury as hypovolemia due to diarrhea and vomiting, adrenal insufficiency or renal salt wasting. Delay of methotrexate secretion can be due to extravascular fluid accumulation such as ascites, pleural effusion, and intracranial fluid (3). In the administration of methotrexate, we should pay attention to the patient's concurrent medications. Because some drugs increase the half-life of methotrexate and the risk of toxicity like Indomethacin, ketoprofen, and naproxen (4). On the other hand, over $50 \%$ of MTX is bound to albumin and simultaneous ingestion of Non-steroid anti-rheumatic

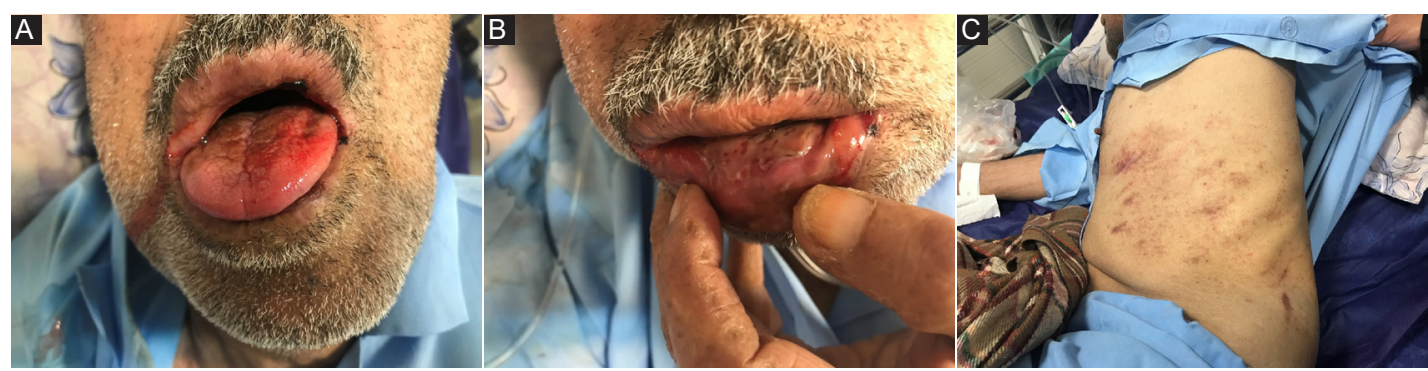

Figure 2. (A) Mucocutaneous, (B) mucocutaneous and (C) cutaneous lesions in case number 2. 
drugs, sulfonamides, retinoids, tetracyclines and salicylates increases the risk of methotrexate poisoning (5). The liver also plays a part in the clearance of methotrexate. Hepatotoxicity is classified based on elevated liver enzymes. Hepatotoxicity after high dose methotrexate is less common than continuous and oral administration of methotrexate (6).

Nausea and vomiting are some of the side effects of methotrexate (7). Hypovolemia is common in old age due to lack of water consumption. All of these can cause dehydration. Therefore, methotrexate secretion is disrupted by renal tubules and increases the risk of methotrexate poisoning (8). So, one way to prevent poisoning is to educate the patient about adequate fluid and water consumption. In our case, nausea and vomiting may have caused the poisoning within 10 days.

In the study conducted by Elmorsi et al, the interaction of methotrexate drug with nonsteroidal anti-inflammatory drugs has been reported (4). Use of Non-steroidal antiinflammatory drugs is common in old age due to chronic debilitating diseases. Therefore, it is important for the physician to pay attention to the simultaneous use of other patient medications, especially over-the-counter (OTC) drugs. Our patient also took indomethacin along with methotrexate.

The liver also plays a role in the excretion of methotrexate (5). Use of folic acid or folinic acid is associated with a reduced incidence of aminotransferases elevation (9). Regular measurement of liver enzymes is necessary for the long-term use of methotrexate. One of our patients also had elevated liver enzymes.

\section{Conclusion}

The prevalence of autoimmune diseases is increasing and consequently the use of immunosuppressive drugs has increased. One of the long-term treatments with harmful side effects is MTX. A significant proportion of methotrexate users are the elderly and illiterate people. One of the important ways to prevent the toxicity of methotrexate is learning how to use it correctly. Training should be provided by the prescribing physician. The pharmacist will re-instruct the patient how to use the drug in the pharmacy correctly. In elderly and illiterate people, education of patient companions is an important issue that can prevent drug poisoning. Medications that are taken weekly should specifically mention the day of use. In cases where the patient has multiple referrals to the physician, and the patient's medication is renewed each time, the physician should recheck the patient's medication at each visit.

\section{Authors' contributions}

Both patients were introduced by HR as a professor. MZL followed the patients as an assistant. Both authors collaborated to gather data and report patients. Both authors read and approved the final manuscript.

\section{Ethical issues}

Informed consent was obtained from patients concerning publication of their information.

\section{Acknowledgement}

The authors are grateful to the participants, their parents, and hospital staff of Emam Reza hospital, Mashhad, Iran, for their cooperation.

\section{References}

1. Singh A, Handa AC. Medication error- a case report of misadventure with methotrexate. JNMA J Nepal Med Assoc 2018; 56(211): 711-5.

2. Feinsilber D, Leoni RJ, Siripala D, Leuck J, Mears KA. Evaluation, identification, and management of acute methotrexate toxicity in high-dose methotrexate administration in hematologic malignancies. Cureus 2018; 10(1): e2040. doi: 10.7759/cureus.2040.

3. Howard SC, McCormick J, Pui CH, Buddington RK, Harvey RD. Preventing and managing toxicities of highdose methotrexate. Oncologist 2016; 21(12): 1471-82. doi: 10.1634/theoncologist.2015-0164.

4. Elmorsi YM, El-Haggar SM, Ibrahim OM, Mabrouk MM. Effect of ketoprofen and indomethacin on methotrexate pharmacokinetics in mice plasma and tumor tissues. Eur J Drug Metab Pharmacokinet 2013; 38(1): 27-32. doi: 10.1007/s13318-012-0113-x

5. Knoll K, Anzengruber F, Cozzio A, French LE, Murer C, Navarini AA. Mucocutaneous ulcerations and pancytopenia due to methotrexate overdose. Case Rep Dermatol 2016; 8(3): 287-93. doi: 10.1159/000446692.

6. Lindsay K, Fraser AD, Layton A, Goodfield M, Gruss H, Gough A. Liver fibrosis in patients with psoriasis and psoriatic arthritis on long-term, high cumulative dose methotrexate therapy. Rheumatology (Oxford) 2009; 48(5): 569-72. doi: 10.1093/rheumatology/kep023.

7. Gaies E, Jebabli N, Trabelsi S, Salouage I, Charfi R, Lakhal M, et al. Methotrexate side effects: review article. J Drug Metab Toxicol 2012; 3(4): 125. doi: 10.4172/2157-7609.1000125.

8. Widemann BC, Adamson PC. Understanding and managing methotrexate nephrotoxicity. Oncologist 2006; 11(6): 694-703. doi: 10.1634/theoncologist.11-6-694.

9. Prey S, Paul C. Effect of folic or folinic acid supplementation on methotrexate-associated safety and efficacy in inflammatory disease: a systematic review. Br J Dermatol 2009; 160(3): 622-8. doi: 10.1111/j.1365-2133.2008.08876.x. 